\title{
Sharpness of Muqattash-Yahdi problem
}

\author{
CHAO-PING $\mathrm{CHEN}^{1}$ and CRISTINEL MORTICI ${ }^{2 *}$ \\ ${ }^{1}$ School of Mathematics and Informatics, Henan Polytechnic University, \\ Jiaozuo City 454003, Henan Province, People's Republic of China \\ ${ }^{2}$ Valahia University of Târgovişte, Department of Mathematics,
}

Bd. Unirii 18, 130082 Târgovişte, Romania

E-mail: chenchaoping@sohu.com / cmortici@valahia.ro

\begin{abstract}
Let $\psi$ denote the psi (or digamma) function. We determine the values of the parameters $p, q$ and $r$ such that$$
\psi(n) \approx \ln (n+p)-\frac{q}{n+r}
$$

is the best approximations. Also, we present closer bounds for psi function, which sharpens some known results due to Muqattash and Yahdi, Qi and Guo, and Mortici.
\end{abstract}

Mathematical subject classification: 33B15, 26D15.

Key words: psi function, polygamma functions, rate of convergence, approximations.

The gamma function is usually defined for $x>0$ by

$$
\Gamma(x)=\int_{0}^{\infty} t^{x-1} e^{-t} d t .
$$

The logarithmic derivative of the gamma function:

$$
\psi(x)=\frac{d}{d x}\{\ln \Gamma(x)\}=\frac{\Gamma^{\prime}(x)}{\Gamma(x)} \quad \text { or } \quad \ln \Gamma(x)=\int_{1}^{x} \psi(t) d t
$$

\#CAM-308/10. Received: 21/XII/10. Accepted: 24/VIII/11.

*Corresponding author. 
is known as the psi (or digamma) function. The successive derivatives of the psi function $\psi(x)$ :

$$
\psi^{(n)}(x):=\frac{d^{n}}{d x^{n}}\{\psi(x)\} \quad(n \in \mathbb{N})
$$

are called the polygamma functions.

The following asymptotic formula is well known for the psi function:

$$
\begin{aligned}
\psi(x) & \sim \ln x-\frac{1}{2 x}-\sum_{n=1}^{\infty} \frac{B_{2 n}}{2 n x^{2 n}} \\
& =\ln x-\frac{1}{2 x}-\frac{1}{12 x^{2}}+\frac{1}{120 x^{4}}-\frac{1}{252 x^{6}}+\cdots \quad(x \rightarrow \infty)
\end{aligned}
$$

(see [1, p. 259]), where

$$
\begin{aligned}
& B_{0}=1, \quad B_{1}=-\frac{1}{2}, \quad B_{2}=\frac{1}{6}, \quad B_{4}=-\frac{1}{30}, \quad B_{6}=\frac{1}{42}, \\
& B_{8}=-\frac{1}{30}, \quad B_{10}=\frac{5}{66}, \ldots, \quad \text { and } \quad B_{2 n+1}=0 \quad(n \in \mathbb{N})
\end{aligned}
$$

are the Bernoulli numbers.

Recently, the approximations of the following form:

$$
\psi(x) \approx \ln (x+a)-\frac{1}{x}, \quad a \in[0,1], \quad x \in[2, \infty)
$$

were studied by Muqattash and Yahdi [6]. They computed the error

$$
\left|\psi(x)-\left(\ln (x+a)-\frac{1}{x}\right)\right| \leq \ln \left(1+\frac{1}{x}\right) \leq \ln \left(\frac{3}{2}\right)=0.4054651081 \ldots,
$$

and then the approximation (2) was compared with the approximation obtained by considering the first two terms of the series (1), that is

$$
\left|\psi(x)-\left(\ln (x+a)-\frac{1}{x}\right)\right| \leq\left|\psi(x)-\left(\ln (x)-\frac{1}{2 x}\right)\right| .
$$

Very recently, the family (2) was also discussed by Qi and Guo [7]. One of their main results is the following inequality on $x \in(0, \infty)$ :

$$
\ln \left(x+\frac{1}{2}\right)-\frac{1}{x}<\psi(x)<\ln \left(x+e^{-\gamma}\right)-\frac{1}{x},
$$

where $\gamma=0.577215 \ldots$ is the Euler-Mascheroni constant. 
In the final part of the paper [6], the authors wonder whether there are profitable constants $a \in[0,1]$ and $b \in[1,2]$ for which better approximations of the form

$$
\psi(x) \approx \ln (x+a)-\frac{1}{b x}
$$

can be obtained. Mortici [3] solved this open problem and proved that the best approximations (4) appear for

$$
a=\frac{1}{\sqrt{6}}, \quad b=6-2 \sqrt{6}
$$

and

$$
a=-\frac{1}{\sqrt{6}}, \quad b=6+2 \sqrt{6} .
$$

Moreover, the author derived from [3, Theorem 2.1] the following symmetric double inequality: For $x>\frac{1}{\sqrt{6}}=0.40824829 \ldots$,

$$
\ln \left(x-\frac{1}{\sqrt{6}}\right)-\frac{1}{(6+2 \sqrt{6}) x} \leq \psi(x) \leq \ln \left(x+\frac{1}{\sqrt{6}}\right)-\frac{1}{(6-2 \sqrt{6}) x} .
$$

This double inequality is more accurate than the estimations (3) of Qi and Guo.

We define the sequence $\left(v_{n}\right)_{n \in \mathbb{N}}$ by

$$
v_{n}=\psi(n)-\left(\ln (n+p)-\frac{q}{n+r}\right) .
$$

We are interested in finding the values of the parameters $p, q$ and $r$ such that $\left(v_{n}\right)_{n \in \mathbb{N}}$ is the fastest sequence which would converge to zero. This provides the best approximations of the form:

$$
\psi(n) \approx \ln (n+p)-\frac{q}{n+r} .
$$

Our study is based on the following Lemma 1, which provides a method for measuring the speed of convergence.

Lemma 1 (see [4] and [5]). If the sequence $\left(\lambda_{n}\right)_{n \in \mathbb{N}}$ converges to zero and if there exists the following limit:

$$
\lim _{n \rightarrow \infty} n^{k}\left(\lambda_{n}-\lambda_{n+1}\right)=l \in \mathbb{R} \quad(k>1),
$$


then

$$
\lim _{n \rightarrow \infty} n^{k-1} \lambda_{n}=\frac{l}{k-1} \quad(k>1) .
$$

Theorem 1. Let the sequence $\left(v_{n}\right)_{n \in \mathbb{N}}$ be defined by (6). Then for

$$
\left\{\begin{array}{l}
p=-\frac{1}{2}-\frac{1}{6} \sqrt{9+6 \sqrt{3}} \\
q=-\frac{1}{6} \sqrt{9+6 \sqrt{3}} \\
r=-\frac{1}{2}-\frac{1}{18} \sqrt{3} \sqrt{9+6 \sqrt{3}}
\end{array}\right.
$$

or

$$
\left\{\begin{array}{l}
p=-\frac{1}{2}+\frac{1}{6} \sqrt{9+6 \sqrt{3}} \\
q=\frac{1}{6} \sqrt{9+6 \sqrt{3}} \\
r=-\frac{1}{2}+\frac{1}{18} \sqrt{3} \sqrt{9+6 \sqrt{3}}
\end{array}\right.
$$

we have

$$
\lim _{n \rightarrow \infty} n^{5}\left(v_{n}-v_{n+1}\right)=\frac{1}{180}+\frac{\sqrt{3}}{54} \text { and } \lim _{n \rightarrow \infty} n^{4} v_{n}=\frac{1}{720}+\frac{\sqrt{3}}{216} .
$$

The speed of convergence of the sequence $\left(v_{n}\right)_{n \in \mathbb{N}}$ is given by the order estimate $O\left(n^{-4}\right)$ as $n \rightarrow \infty$.

Proof. First of all, we write the difference $v_{n}-v_{n+1}$ as the following power series in $n^{-1}$ :

$$
\begin{aligned}
& v_{n}-v_{n+1} \\
& =\frac{2 q-2 p-1}{2 n^{2}}+\frac{-3 q-6 q r+3 p+3 p^{2}+1}{3 n^{3}} \\
& \quad+\frac{4 q+12 q r+12 q r^{2}-1-4 p-6 p^{2}-4 p^{3}}{4 n^{4}} \\
& +\frac{-5 q-20 q r-30 q r^{2}-20 q r^{3}+1+5 p+10 p^{2}+10 p^{3}+5 p^{4}}{5 n^{5}} \\
& +O\left(\frac{1}{n^{6}}\right) \quad(n \rightarrow \infty) .
\end{aligned}
$$


According to Lemma 1, the three parameters $p, q$ and $r$, which produce the fastest convergence of the sequence $\left(v_{n}\right)_{n \in \mathbb{N}}$ are given by (10)

$$
\left\{\begin{array}{l}
2 q-2 p-1=0 \\
-3 q-6 q r+3 p+3 p^{2}+1=0 \\
4 q+12 q r+12 q r^{2}-1-4 p-6 p^{2}-4 p^{3}=0,
\end{array}\right.
$$

that is, by (8) and (9). We thus find that

$$
v_{n}-v_{n+1}=\left(\frac{1}{180}+\frac{\sqrt{3}}{54}\right) \frac{1}{n^{5}}+O\left(\frac{1}{n^{6}}\right) \quad(n \rightarrow \infty) .
$$

Finally, by using Lemma 1, we obtain the assertion (1) of Theorem 1.

Solutions (8) and (9) provide the best approximations of type (7):

$$
\psi(n) \approx \ln \left(n-\frac{1}{2}-\frac{1}{6} \sqrt{9+6 \sqrt{3}}\right)+\frac{3 \sqrt{9+6 \sqrt{3}}}{18 n-9-\sqrt{3} \sqrt{9+6 \sqrt{3}}}
$$

and

$$
\psi(n) \approx \ln \left(n-\frac{1}{2}+\frac{1}{6} \sqrt{9+6 \sqrt{3}}\right)-\frac{3 \sqrt{9+6 \sqrt{3}}}{18 n-9+\sqrt{3} \sqrt{9+6 \sqrt{3}}} .
$$

Theorem 2 below presents closer bounds for psi function.

Theorem 2. For $x>\frac{1}{2}+\frac{1}{6} \sqrt{9+6 \sqrt{3}}=1.23394491 \ldots$, then

$$
\begin{aligned}
\ln (x & \left.-\frac{1}{2}-\frac{1}{6} \sqrt{9+6 \sqrt{3}}\right)+\frac{3 \sqrt{9+6 \sqrt{3}}}{18 x-9-\sqrt{3} \sqrt{9+6 \sqrt{3}}} \\
& +\left(\frac{1}{720}+\frac{\sqrt{3}}{216}\right) \frac{1}{x^{4}}<\psi(x)<\ln \left(x-\frac{1}{2}+\frac{1}{6} \sqrt{9+6 \sqrt{3}}\right) \\
& -\frac{3 \sqrt{9+6 \sqrt{3}}}{18 x-9+\sqrt{3} \sqrt{9+6 \sqrt{3}}}+\left(\frac{1}{720}+\frac{\sqrt{3}}{216}\right) \frac{1}{x^{4}} .
\end{aligned}
$$


Proof. The lower bound of (13) is obtained by considering the function $F$ defined by

$$
\begin{aligned}
F(x)= & \psi(x)-\ln \left(x-\frac{1}{2}-\frac{1}{6} \sqrt{9+6 \sqrt{3}}\right)-\frac{3 \sqrt{9+6 \sqrt{3}}}{18 x-9-\sqrt{3} \sqrt{9+6 \sqrt{3}}} \\
& -\left(\frac{1}{720}+\frac{\sqrt{3}}{216}\right) \frac{1}{x^{4}}, \quad x>\frac{1}{2}+\frac{1}{6} \sqrt{9+6 \sqrt{3}} .
\end{aligned}
$$

We conclude from the asymptotic formula (1) that

$$
\lim _{x \rightarrow \infty} F(x)=0 .
$$

It follows form [2, Theorem 9] that

$$
\begin{aligned}
\frac{1}{x}+\frac{1}{2 x^{2}}+ & \frac{1}{6 x^{3}}-\frac{1}{30 x^{5}}<\psi^{\prime}(x) \\
-\frac{1}{30 x^{5}}+\frac{1}{4}+\frac{1}{42 x^{7}}, \quad x & >0 .
\end{aligned}
$$

Differentiating $F(x)$ with respect to $x$ and applying the second inequality in (14) yields, for $x>\frac{1}{2}+\frac{1}{6} \sqrt{9+6 \sqrt{3}}$,

$$
\begin{aligned}
F^{\prime}(x)= & \psi^{\prime}(x)-\frac{6}{6 x-3-\sqrt{9+6 \sqrt{3}}}+\frac{54 \sqrt{9+6 \sqrt{3}}}{(18 x-9-\sqrt{3} \sqrt{9+6 \sqrt{3}})^{2}} \\
& +\frac{10 \sqrt{3}+3}{540 x^{5}}<\frac{1}{x}+\frac{1}{2 x^{2}}+\frac{1}{6 x^{3}}-\frac{1}{30 x^{5}}+\frac{1}{42 x^{7}} \\
& -\frac{6}{6 x-3-\sqrt{9+6 \sqrt{3}}}+\frac{54 \sqrt{9+6 \sqrt{3}}}{(18 x-9-\sqrt{3} \sqrt{9+6 \sqrt{3}})^{2}}+\frac{10 \sqrt{3}+3}{540 x^{5}} \\
= & -\frac{P(x)}{7 x^{7}(6 x-3-\sqrt{9+6 \sqrt{3}})(18 x-9-\sqrt{3} \sqrt{9+6 \sqrt{3}})^{2}},
\end{aligned}
$$

where

$$
\begin{aligned}
P(x)= & 312+96 \sqrt{9+6 \sqrt{3}} \sqrt{3}+426 \sqrt{3}+151 \sqrt{9+6 \sqrt{3}} \\
& +(593 \sqrt{9+6 \sqrt{3}}+1902 \sqrt{3}+1113+372 \sqrt{9+6 \sqrt{3}} \sqrt{3})(x-1)
\end{aligned}
$$




$$
\begin{aligned}
& +(540 \sqrt{9+6 \sqrt{3}} \sqrt{3}+880 \sqrt{9+6 \sqrt{3}}+1425+3192 \sqrt{3})(x-1)^{2} \\
& +(567 \sqrt{9+6 \sqrt{3}}+336 \sqrt{9+6 \sqrt{3}} \sqrt{3}+705+2310 \sqrt{3})(x-1)^{3} \\
& +(630 \sqrt{3}+84 \sqrt{9+6 \sqrt{3}} \sqrt{3}+189+147 \sqrt{9+6 \sqrt{3}})(x-1)^{4} \\
& >0 \text { for } x>\frac{1}{2}+\frac{1}{6} \sqrt{9+6 \sqrt{3}} .
\end{aligned}
$$

Therefore, $F^{\prime}(x)<0$ for $x>\frac{1}{2}+\frac{1}{6} \sqrt{9+6 \sqrt{3}}$. This leads to

$$
F(x)>\lim _{x \rightarrow \infty} F(x)=0 .
$$

This means that the first inequality in (13) holds for $x>\frac{1}{2}+\frac{1}{6} \sqrt{9+6 \sqrt{3}}$.

The upper bound of (13) is obtained by considering the function $G$ defined by

$$
\begin{aligned}
G(x)= & \psi(x)-\ln \left(x-\frac{1}{2}+\frac{1}{6} \sqrt{9+6 \sqrt{3}}\right)+\frac{3 \sqrt{9+6 \sqrt{3}}}{18 x-9+\sqrt{3} \sqrt{9+6 \sqrt{3}}} \\
& -\left(\frac{1}{720}+\frac{\sqrt{3}}{216}\right) \frac{1}{x^{4}}, \quad x>0 .
\end{aligned}
$$

We conclude from the asymptotic formula (1) that

$$
\lim _{x \rightarrow \infty} G(x)=0 .
$$

Differentiating $G(x)$ with respect to $x$ and applying the first inequality in (14) yields, for $x>0$,

$$
\begin{aligned}
G^{\prime}(x)= & \psi^{\prime}(x)-\frac{6}{6 x-3+\sqrt{9+6 \sqrt{3}}}-\frac{54 \sqrt{9+6 \sqrt{3}}}{(18 x-9+\sqrt{3} \sqrt{9+6 \sqrt{3}})^{2}} \\
& +\frac{10 \sqrt{3}+3}{540 x^{5}}>\frac{1}{x}+\frac{1}{2 x^{2}}+\frac{1}{6 x^{3}}-\frac{1}{30 x^{5}}-\frac{6}{6 x-3+\sqrt{9+6 \sqrt{3}}} \\
& -\frac{54 \sqrt{9+6 \sqrt{3}}}{(18 x-9+\sqrt{3} \sqrt{9+6 \sqrt{3}})^{2}}+\frac{10 \sqrt{3}+3}{540 x^{5}} \\
= & \frac{Q(x)}{x^{5}(6 x-3+\sqrt{9+6 \sqrt{3}})(18 x-9+\sqrt{3} \sqrt{9+6 \sqrt{3}})^{2}},
\end{aligned}
$$


where

$$
\begin{aligned}
Q(x)= & (-90 \sqrt{3}+21 \sqrt{9+6 \sqrt{3}}-27+12 \sqrt{3} \sqrt{9+6 \sqrt{3}}) x^{2} \\
& +(30 \sqrt{3}-39-3 \sqrt{9+6 \sqrt{3}}) x+\sqrt{9+6 \sqrt{3}}-6 \sqrt{3}+6 \\
= & (-90 \sqrt{3}+21 \sqrt{9+6 \sqrt{3}}-27+12 \sqrt{3} \sqrt{9+6 \sqrt{3}}) \\
& \times\left(x-x_{1}\right)\left(x-x_{2}\right)
\end{aligned}
$$

with

$$
\begin{aligned}
x_{1}= & \frac{13-10 \sqrt{3}+\sqrt{9+6 \sqrt{3}}}{2(-30 \sqrt{3}+7 \sqrt{9+6 \sqrt{3}}-9+4 \sqrt{3} \sqrt{9+6 \sqrt{3}})} \\
= & 0.0638967475 \ldots, \\
x_{2}= & \frac{13-10 \sqrt{3}+\sqrt{9+6 \sqrt{3}}}{2(-30 \sqrt{3}+7 \sqrt{9+6 \sqrt{3}}-9+4 \sqrt{3} \sqrt{9+6 \sqrt{3}})} \\
= & 0.158650823 \ldots
\end{aligned}
$$

Therefore, $Q(x)>0$ and $G^{\prime}(x)>0$ for $x>x_{2}$. This leads to

$$
G(x)<\lim _{x \rightarrow \infty} G(x)=0 \quad x>x_{2} .
$$

This means that the second inequality in (13) holds for $x>0.158650823 \ldots$.

Some computer experiments indicate that for $x>2.30488055$, the lower bound in (13) is sharper than one in (5). For $x>0.5690291018$, the upper bound in (13) is sharper than one in (5).

The inequality (13) provides the best approximations:

$$
\psi(x) \approx \ln \left(x-\frac{1}{2}-\frac{1}{6} \sqrt{9+6 \sqrt{3}}\right)+\frac{3 \sqrt{9+6 \sqrt{3}}}{18 x-9-\sqrt{3} \sqrt{9+6 \sqrt{3}}}
$$


and

$$
\psi(x) \approx \ln \left(x-\frac{1}{2}+\frac{1}{6} \sqrt{9+6 \sqrt{3}}\right)-\frac{3 \sqrt{9+6 \sqrt{3}}}{18 x-9+\sqrt{3} \sqrt{9+6 \sqrt{3}}} .
$$

Acknowledgements. The work of the second author was supported by a grant of the Romanian National Authority for Scientific Research, CNCS UEFISCDI, project number PN-II-ID-PCE-2011-3-0087.

\section{REFERENCES}

[1] M. Abramowitz and I.A. Stegun (Editors), Handbook of Mathematical Functions with Formulas, Graphs, and Mathematical Tables. Applied Mathematics Series, 55, Ninth printing, National Bureau of Standards, Washington, D.C. (1972).

[2] H. Alzer, On some inequalities for the gamma and psi functions. Math. Comp., 66 (1997), 373-389.

[3] C. Mortici, The proof of Muqattash-Yahdi conjecture. Math. Comput. Modelling, 51 (2010), 1154-1159.

[4] C. Mortici, New approximations of the gamma function in terms of the digamma function. Appl. Math. Lett., 23 (2010), 97-100.

[5] C. Mortici, Product approximations via asymptotic integration. Amer. Math. Monthly, 117 (2010), 434-441.

[6] I. Muqattash and M. Yahdi, Infinite family of approximations of the digamma function. Math. Comput. Modelling, 43 (2006), 1329-1336.

[7] F. Qi and B.-N. Guo, Sharp inequalities for the psi function and harmonic numbers, arXiv:0902.2524v1 [math CA]. 